Decision Support

\title{
A method for member selection of cross-functional teams using the individual and collaborative performances
}

\author{
Bo Feng ${ }^{\mathrm{a}, \mathrm{b}}$, Zhong-Zhong Jiang ${ }^{\mathrm{a}}$, Zhi-Ping Fan ${ }^{\mathrm{a}, *}, \mathrm{Na} \mathrm{Fu}^{\mathrm{c}}$ \\ a Department of Management Science and Engineering, School of Business Administration, Northeastern University, Shenyang 110004, China \\ ${ }^{\mathrm{b}}$ Department of Decision Science, School of Business Administration, South China University of Technology, Guangzhou 510640, China \\ ${ }^{\mathrm{c}}$ Business School, Dublin City University, Dublin 9, Ireland
}

\section{A R T I C L E I N F O}

\section{Article history:}

Received 16 July 2008

Accepted 27 August 2009

Available online 2 September 2009

\section{Keywords:}

Cross-functional team (CFT)

Member selection

Individual and collaborative performances

Multi-objective 0-1 programming

Nondominated sorting genetic algorithm II

(NSGA-II)

\begin{abstract}
A B S T R A C T
The member selection problem is an important aspect of the formation of cross-functional teams (CFTs). Selecting suitable members from a set of candidates will facilitate the successful task accomplishment. In the existing studies of member selection, the individual performance concerning a single candidate is mostly used, whereas the collaborative performance associating with a pair of candidates is overlooked. In this paper, as a solution to this problem, we propose a method for member selection of CFTs, where both the individual performance of candidates and the collaborative performance between candidates are considered. In order to select the desired members, firstly, a multi-objective $0-1$ programming model is built using the individual and collaborative performances, which is an NP-hard problem. To solve the model, we develop an improved nondominated sorting genetic algorithm II (INSGA-II). Furthermore, a real example is employed to illustrate the suitability of the proposed method. Additionally, extensive computational experiments to compare INSGA-II with the nondominated sorting genetic algorithm II (NSGA-II) are conducted and much better performance of INSGA-II is observed.
\end{abstract}

(C) 2009 Elsevier B.V. All rights reserved.

\section{Introduction}

Cross-functional team (CFT), sometimes called a multidisciplinary team, consists of members from different functional areas such as engineering, manufacturing or marketing (Keller, 2001; Fitzpatrick and Askin, 2005), or from various research disciplines such as chemistry, electronics or biomaterial. Organizations are increasingly using CFTs to address new product development (NPD) (Holand et al., 2000; Dowlatshahi, 1999; Wang et al., 2003; Chen and Lin, 2004). By the use of cross-functional teams (CFTs), organizations attempt to improve coordination and integration (Ford and Randolph, 1992), span organizational boundaries (Ancona, 1990), improve timing of technology developments and reduce uncertainty levels (Hitt et al., 1993).

In the development of CFTs, team formation is the first stage. In recent years, the formation of CFTs has gained increasing attention (Zakarian and Kusiak, 1999; Wang et al., 2003; Chen and Lin, 2004; van de Water et al., 2007). In order to ensure the success of CFTs, it is important to choose the team members carefully with desirable qualifications to avoid extra time and undesirable budget consumed (Chen and Lin, 2004).

\footnotetext{
* Corresponding author. Tel.: +86 248368 7753; fax: +86 2423891569. E-mail addresses: neu_fengbo@163.com (B. Feng), zpfan@mail.neu.edu.cn (Z.-P. Fan).
}

In the existing research of the formation of CFTs, a number of scholars have discussed the qualification or characteristics of desired members. Wang et al. (2003) discuss the requirements for desired members, such as creation ability, management ability, utilization rates, cooperation levels, etc. Chen and Lin (2004) regard the knowledge of multiple functional areas, good teamwork skills and good working relationship as important evaluation criteria for member selection of CTFs. To complement team members' strength and weakness, Haque et al. (2000) state that good teams should be composed of different and specialized members with different values, interests and capabilities. Fitzpatrick and Askin (2005) point out that the formation of teams requires consideration of innate tendencies, interpersonal skills and technical skills. In addition, it is commonly evident that communication of team members has positive effects on team performance (Campion et al., 1993).

Existing research focuses on the individual performance of candidates, while it seldom incorporates the collaborative performance between candidates in member selection of CFTs. In a CFT, members have to transcend traditional departmental boundaries onto a common platform to share their ideas and concerns. Thus collaborative innovation in product development projects becomes an emerging challenge in innovation management (Cowan et al., 2007; Schilling and Phelps, 2007; Emden et al., 2006; Fan et al., 2009; Hacklin et al., 2006). Collaboration among members of CFTs plays a significant role since the performance of a CFT 
depends on the good combination of information and expertise of all team members. Good collaboration relationships (Chen and Lin, 2004) among members may promote favorable interpersonal interaction, cohesiveness, member satisfaction, mutual understanding and trust (Msanjila and Afsarmanesh, 2008), reduce uncertainty and conflicts, shorten adopting duration, and lead to higher team performance in the future (Gloor et al., 2008). As for the collaborative relationship, some researchers have argued that firms favor past cooperators when choosing team members (Kaihara and Fujii, 2008), which can reduce uncertainty regarding potential members' capabilities and reliabilities (Balakrishnan and Koza, 1993; Gulati, 1998). Gulati (1998) finds that in North America, the longer the prior history between two firms, the more likely they are to recreate collaborations. This manifests that the cooperators prefer the partners with whom they have successful collaboration before.

Furthermore, utilizing quantitative methods for the formation of CFTs has been a topic of the existing research. Zakarian and Kusiak (1999) propose a method for a team formation based on quality function deployment (QFD) and AHP, where developers' disciplines for product development tasks are considered. Wang et al. (2003) present an integer-programming model to solve the team organization problem in which a heuristic algorithm is used to solve the integer-programming model. Chen and Lin (2004) develop a mathematical model and a five-step approach to support the formation of CFTs. They use AHP to compare different designers according to their competencies and to select the most appropriate members for a team. Fitzpatrick and Askin (2005) develop a mathematical programming method for forming effective worker teams with multi-functional skill requirements.

However, in the existing methods for the formation of CFTs, the individual performance concerning a single candidate is extensively studied, whereas the collaborative performance on a pair of candidates is seldom considered. Thus an appropriate model and an effect algorithm should be investigated to solve the problem of member selection of CFTs using the individual and collaborative performances.

Consequently, the aim of this paper is to propose a novel method for member selection of CFTs, in which both the individual performance of candidates and the collaborative performance between candidates are employed. In doing so, firstly, the problem of member selection of CFTs is described and a conceptual framework for member selection is presented. Then, a multi-objective $0-1$ programming model is built using the individual and collaborative performances, which is an NP-hard problem. In this model, three sub-objectives, including individual performance, interior organizational collaborative performance and exterior organizational collaborative performance, are considered. Additionally, an improved nondominated sorting genetic algorithm II (INSGA-II) is developed to solve the model.

The remainder of this paper is organized as follows: in Section 2 , a conceptual framework is presented to generalize the problem of member selection of CFTs. The mathematical model for member selection of CFTs using the individual and collaborative performances is built in Section 3. In Section 4, an INSGA-II is developed to solve the model. In Section 5, a real example and extensive comparison experiments are presented to show the acapability of the proposed method. Finally, in Section 6, the conclusions of this paper are outlined.

\section{A conceptual framework for member selection of CFTs}

As discussed in Introduction, the problem concerned in this paper is to select several desired members from the candidates belonging to different functional departments (or disciplines) to

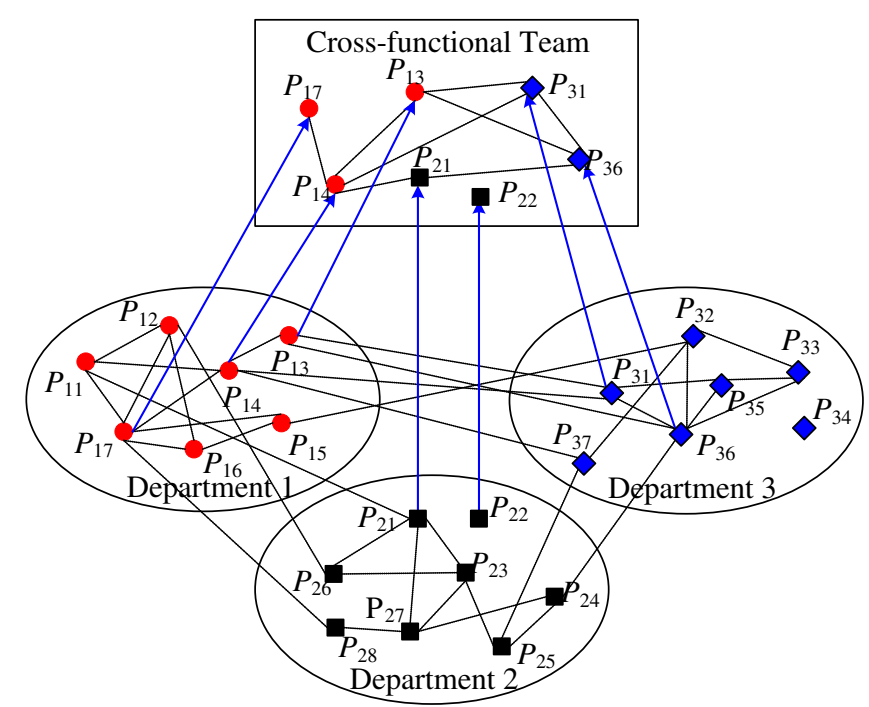

Fig. 1. The formation of a CFT.

form a cross-functional team using the individual and collaborative performances. Some of the candidates have prior collaboration relationship within a department or among departments. Suppose that the number of desired members from each department is not less than one. The CFT composed of selected members has the optimal individual and collaborative performances. Fig. 1 shows the formation of a CFT, i.e., seven members are selected from 22 candidates of 3 departments. In Fig. 1, the distributed candidates and their prior cooperative relationships form a collaborative network. The dashed line '-' denotes the collaborative relationship between candidates. The ellipse ' $\bigcirc$ ' represents the functional department. The figures ' $\boldsymbol{\prime}$ ', ' $\boldsymbol{\nabla}$ ' and ' $\boldsymbol{\nabla}$ ' denote the candidates of departments (or disciplines) 1, 2 and 3, respectively. The top rectangular ' $\square$ ' represents a CFT, and the figures ' $\bullet$ ', ' $\mathbf{\square}$ ' and ' $\boldsymbol{\nabla}$ ' within it denote the desired members selected from departments (or disciplines) 1,2 and 3 , respectively. The arrow line ' $\longrightarrow$ ' denotes that the desired members are selected from the candidates of different departments. Fig. 1 also shows that the formation of a CFT is essential to select a clique (or a cluster) consisted of several desired members from candidates inside or outside the organization. The selected clique has the optimal group performances on the individual and collaborative aspects.

Based on the above analysis, the problem of member selection of CFTs can be generally described by a conceptual framework as shown in Fig. 2. In the conceptual framework, both the individual performance on a single candidate and the collaborative performance on a pair of candidates are considered to select members to form a CFT. Furthermore, sub-objectives can be specified according to the requirements of CFT formation. Different sub-objectives are measured by the corresponding criteria. Also the candidates with collaborative relationships among different departments are shown in the framework. Particularly, in this paper, we divide collaborative performance into two sub-objectives. Consequently, three key sub-objectives are considered, i.e., individual performance, interior organizational collaborative performance and exterior organizational performance.

\section{Model for member selection of CFTs}

\subsection{Notations}

In order to describe the problem of member selection of CFTs, the following notations are used throughout this paper: 


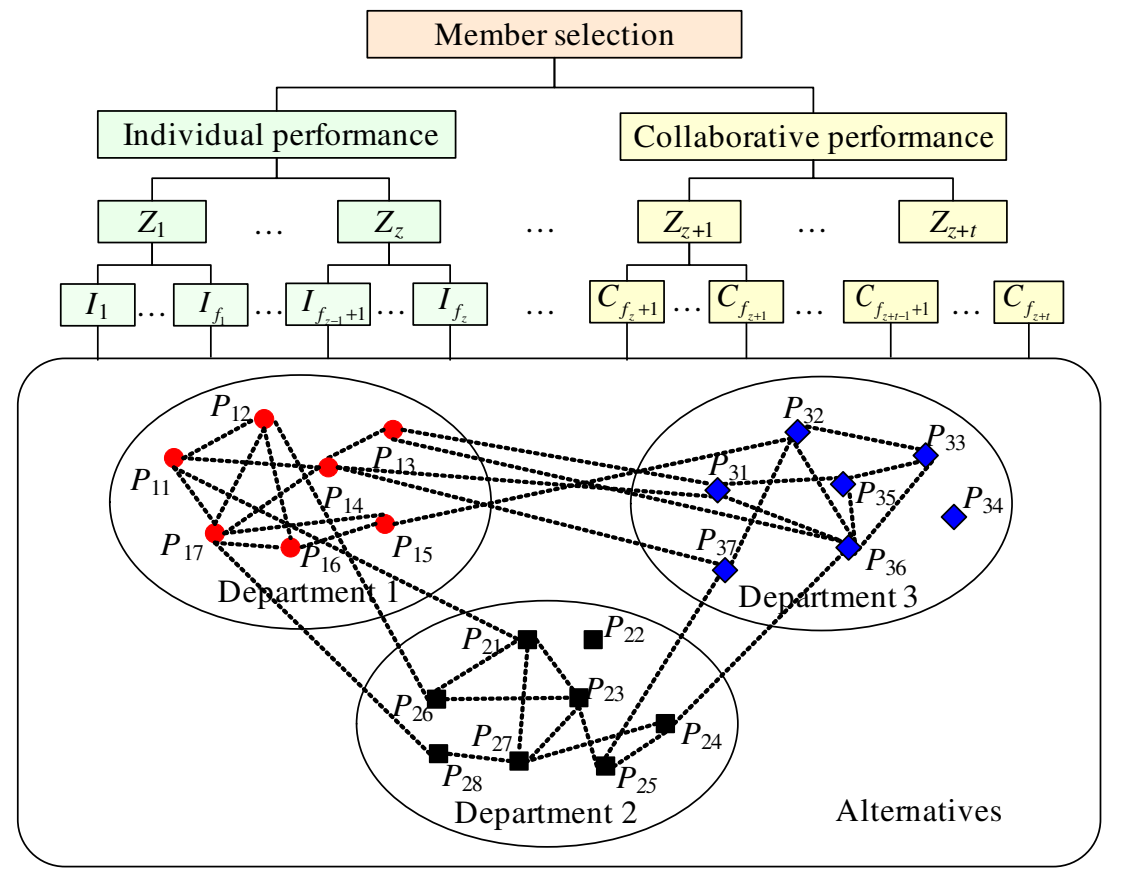

Fig. 2. A conceptual framework for member selection of a CFT.

$P_{i}$

$S_{j}$

$N_{j}$

$I_{g}$

$C_{k}^{(i n)}$

$C^{(e x)}$

$v_{g}$

$w_{k}^{(i n)}$

$x_{i}$ total number of candidates

total number of functional departments

total number of candidates in functional department $j, \sum_{j=1}^{h} n_{j}=n$

total number of desired members to form a CFT

total number of desired members from functional department $j, \sum_{j=1}^{h} q_{j}=q$

total number of criteria for measuring individual performance

total number of criteria for measuring interior organizational collaborative performance

candidate $i, i=1,2, \ldots, n$

set of candidates in functional department $j, j=1,2, \ldots, h$

index set of candidates in set $S_{j}, j=1,2, \ldots, h$

criterion $g$ for measuring the individual performance, $g=1,2, \ldots, l$

criterion $k$ for measuring the interior organizational collaborative performance, $k=1,2, \ldots, m$

the criterion for measuring the exterior organizational collaborative performance

weight of criterion $I_{g}, \sum_{g=1}^{l} v_{g}=1,0 \leqslant v_{g} \leqslant 1$ and $g=1$, $2, \ldots, l$

weight of criterion $C_{k}^{(i n)}, \sum_{k=1}^{m} w_{k}^{(i n)}=1,0 \leqslant w_{k}^{(i n)} \leqslant 1$ and $k=1,2, \ldots, m$

$0-1$ variable, $x_{i}=1$ if candidate $P_{i}$ is selected; $x_{i}=0$, otherwise

\subsection{Individual performance}

Let $D=\left[d_{i g}\right]_{n \times l}$ be the decision matrix on individual performance, where $d_{i g}$ is the consequence with a numerical value of candidate $P_{i}$ with respect to criterion $I_{g}$. In the real world, criteria for measuring individual performance could be work experience, specialized knowledge, functional expertise, etc. The criteria can be finalized according to the requirement of a project task. If criterion $I_{g}$ is objective, then the corresponding criterion value can be obtained according to the statistic data or resorting on the measure instrument. If criterion $I_{g}$ is subjective, then the corresponding criterion value can be obtained by experts' assessment using scores from 1 to 10 (1: very bad, 10: very good). The above measure instrument can be also applied to the measurement of the criteria on collaborative performance.

Regarding commensurability between various criteria, the normalization of criterion values is commonly used. According to the approach proposed by Hwang and Yoon (1981), every element in matrix $D=\left[d_{i g}\right]_{n \times l}$ is normalized into a corresponding element in matrix $D^{\prime}=\left[d_{i g}^{\prime}\right]_{n \times l}$ using the following formulas:

$d_{i g}^{\prime}=\frac{d_{i g}-d_{g}^{\min }}{d_{g}^{\max }-d_{g}^{\min }}, \quad i=1,2, \ldots, n ;$

$g=1,2, \ldots, l$, for benefit criteria,

$d_{i g}^{\prime}=\frac{d_{g}^{\max }-d_{i g}}{d_{g}^{\max }-d_{g}^{\min }}, \quad i=1,2, \ldots, n ;$

$g=1,2, \ldots, l$, for cost criteria,

where $d_{g}^{\max }=\max \left\{d_{i g} \mid i=1,2, \ldots, n\right\}, d_{g}^{\min }=\min \left\{d_{i g} \mid i=1,2, \ldots, n\right\}$, $g=1,2, \ldots, l$.

Suppose the decision maker gives criterion weights $v_{g}, g=$ $1,2, \ldots, l$, by direct assignment or using AHP (see Saaty, 1980). To highlight the sub-objective, the utility values of the criteria are synthesized based on multiple criterion decision making (MCDM) theory (Hwang and Yoon, 1981) and multi-criteria utility theory (MCUT) (Figueira et al., 2005). In MCDM theory, if the criteria are independent and additive, then the overall utility value of each candidate could be obtained through weighted aggregation of criterion values (Hwang and Yoon, 1981; Ma et al., 1999; Fan et al., 2002, 2006). By simple additive weighting method (Hwang and Yoon, 1981), the overall value of individual performance of candidate $P_{i}$ can be obtained by

$\varphi_{i}=\sum_{g=1}^{l} v_{g} d_{i g}^{\prime}, \quad i=1,2, \ldots, n$.

According to the overall values of individual performance, $\varphi_{1}, \varphi_{2}, \ldots, \varphi_{n}$, the following optimization model is built to select the most preferred $q$ members from $n$ candidates in $h$ departments only considering individual performance. 
Maximize $Z_{1}=\sum_{i=1}^{n} \varphi_{i} x_{i}$,

subject to $\sum_{i \in N_{j}} x_{i}=q_{j}, \quad j=1,2, \ldots, h$,

$$
x_{i}=1 \text { or } 0, \quad i=1,2, \ldots, n \text {. }
$$

\subsection{Interior organizational collaborative performance}

Let $A^{(i n) k}=\left[a_{i j}^{(i n) k}\right]$ be the decision matrix on interior organizational collaborative performance, where $a_{i j}^{(i n) k}(i \neq j)$ is the numerical consequence that represents the collaboration level between candidates $P_{i}$ and $P_{j}$ with respect to collaborative criterion $C_{k}^{(i n)}$. In the real world, collaborative criteria could be cooperation research performance, communication, knowledge sharing, complementary knowledge base, compatible culture, mutual trust and so on. The collaborative criteria can be determined by the decision maker according to the real requirements of the formation of CFTs. Ordinarily, as for collaborative criteria, we allow nonreciprocal collaboration, i.e., candidate $P_{i}$ gives help to candidate $P_{j}$, but does not receive the help from $P_{j}$ (Nosratinia and Hunter, 2007). That is to say, with respect to some collaborative criterion $C_{k}^{(i n)}$, the consequence that candidate $P_{i}$ collaborates with $P_{j}$ is not the same as the one that candidate $P_{j}$ collaborates with $P_{i}$, i.e., $a_{i j}^{(i n) k} \neq a_{j i}^{(i n) k}$. Let $a_{i i}^{(i n) k}={ }^{\prime}-1$ represent that the own collaborative performance of member $P_{i}$ is not considered, i.e., the values of principle diagonal elements in matrix $A^{(i n) k}$ are null.

According to the approach proposed by Hwang and Yoon (1981), every element in matrix $A^{(i n) k}=\left[a_{i j}^{(i n) k}\right]_{n \times n}$ is normalized to obtain the $A^{\prime(i n) k}=\left[a_{i j}^{\prime(i n) k}\right]_{n \times n}$ using the following equations:

$$
\begin{aligned}
& a_{i j}^{\prime(i n) k}=\frac{a_{i j}^{(i n) k}-a^{(i n) k \min }}{a^{(i n) k \max }-a^{(i n) k \min }}, \quad i, j=1,2, \ldots, n ; i \neq j ; \\
& k=1,2, \ldots, m \text {, for benefit criteria, } \\
& a_{i j}^{\prime(i n) k}=\frac{a^{(i n) k \max }-a_{i j}^{(i n) k}}{a^{(i n) k \max }-a^{(i n) k \min }}, \quad i, j=1,2, \ldots, n ; i \neq j ; \\
& k=1,2, \ldots, m \text {, for cost criteria, }
\end{aligned}
$$

where $\quad a^{(i n) k \max }=\max \left\{a_{i j}^{(i n) k} \mid i, j=1,2, \ldots, n ; i \neq j\right\}, \quad a^{(i n) k \min }=$ $\min \left\{a_{i j}^{(i n) k} \mid i, j=1,2, \ldots, n ; i \neq j\right\}, k=1,2, \ldots, m$.

Suppose the decision maker provides criterion weights $w_{k}^{(i n)}, k=1,2, \ldots, m$, by direct assignment or using AHP (see Saaty, 1980). By the use of simple additive weighting method (Hwang and Yoon, 1981), the overall value of interior organizational collaborative performance between candidates $P_{i}$ and $P_{j}$ can be obtained as

$\phi_{i j}^{(i n)}=\sum_{k=1}^{m} w_{k}^{(i n)} a_{i j}^{\prime(i n) k}, \quad i, j=1,2, \ldots, n ; i \neq j$.

According to the overall values $\phi_{i j}^{(i n)}, i, j=1,2, \ldots, n(i \neq j)$, the following optimization model is built to select the most preferred $q$ members from $n$ candidates in $h$ departments only considering interior organizational collaborative performance.

$$
\begin{array}{ll}
\text { Maximize } & Z_{2}=\sum_{i=1}^{n} \sum_{\substack{j=1 \\
j \neq i}}^{n} \phi_{i j}^{(i n)} x_{i} x_{j}, \\
\text { subject to } & \sum_{i \in N_{j}} x_{i}=q_{j}, \quad j=1,2, \ldots, h, \\
& x_{i}=1 \text { or } 0, \quad i=1,2, \ldots, n .
\end{array}
$$

In model (10)-(12), the objective is to select $q$ members from $n$ candidates to maximize interior organizational collaborative performance of the selected members. It can be seen that model (10)-(12) is a $0-1$ quadratic programming problem. It is similar to the model of maximum diversity problem (MDP), which has been proven to be an NP-Hard problem (Kuo et al., 1993).

\subsection{Exterior organizational collaborative performance}

The success of CFTs stems from the right combination of interior and exterior resource of organization. Sometime, members who come from the exterior organizations would be also required to accomplish a project task. The collaborative performance between the external candidate and the internal one could not be available in this situation. To remedy such weakness of the external candidates, another sub-objective, the exterior organizational collaborative performance (measured by criterion $C^{(e x)}$ ) is considered. Let $A^{(e x)}=\left(a_{1}^{(e x)}, \quad a_{2}^{(e x)}, \ldots, a_{n}^{(e x)}\right)$ be a vector on exterior organizational collaborative performance, where $a_{i}^{(e x)}$ is the assessment value on exterior organizational collaborative performance of candidate $P_{i}$. Here we consider that the decision maker gives his/her overall assessment on the exterior organizational performance of each candidate according to the prior collaborative records of candidates. The assessment value could be expressed in the form of scores from 1 to 10 (1: very bad, 10: very good). Particularly, assessment values on the candidates who have never been involved in the exterior organizational collaboration is 1 .

For the convenience of the analysis, $a_{i}^{(e x)}$ is normalized by the following equation:

$a_{i}^{(e x)}=\frac{a_{i}^{(e x)}-a^{(e x) \min }}{a^{(e x) \max }-a^{(e x) \min }}, \quad i=1,2, \ldots, n$,

where $a^{(e x) \max }=\max \left\{a_{i}^{(e x)} \mid i=1,2, \ldots, n\right\}, \quad a^{(e x) \min }=\min \left\{a_{i}^{(e x)} \mid i=\right.$ $1,2, \ldots, n\}$.

Based on assessment values $a_{i}^{(e x)}, i=1,2, \ldots, n$, the following optimization model is built to select the most preferred $q$ members from $n$ candidates in $h$ departments only considering exterior organizational collaborative performance.

$$
\begin{array}{ll}
\text { Maximize } & Z_{3}=\sum_{i=1}^{n} a_{i}^{(\text {(ex) }} x_{i}, \\
\text { subject to } & \sum_{i \in N_{j}} x_{i}=q_{j}, \quad j=1,2, \ldots, h, \\
& x_{i}=1 \text { or } 0, \quad i=1,2, \ldots, n .
\end{array}
$$

3.5. Model for member selection using the individual and collaborative performances

To solve the problem of member selection of CFTs using the individual and collaborative performances, we combine model (4)-(6), model (10)-(12) and model (14)-(16) to form the following multi-objective $0-1$ programming model.

$$
\begin{array}{ll}
\text { Maximize } & Z_{1}=\sum_{i=1}^{n} \varphi_{i} x_{i}, \\
\text { Maximize } & Z_{2}=\sum_{i=1}^{n} \sum_{\substack{j=1 \\
j \neq i}}^{n} \phi_{i j}^{(i n)} x_{i} x_{j}, \\
\text { Maximize } & Z_{3}=\sum_{i=1}^{n} a_{i}^{(e x)} x_{i}, \\
\text { subject to } & \sum_{i \in N_{j}} x_{i}=q_{j}, \quad j=1,2, \ldots, h, \\
& x_{i}=1 \text { or } 0, \quad i=1,2, \ldots, n .
\end{array}
$$


As for model (17)-(21), its solution space is a function of parameters $n$ and $q$. Let $\Omega$ denote the number of solutions in the solution space, then there is $\Omega=C_{n_{1}}^{q_{1}} \times C_{n_{2}}^{q_{2}} \times \cdots \times C_{n_{h}}^{q_{h}} \leqslant\left[C_{n / h}^{q / h}\right]^{h}=$ $\left\{\frac{(n / h) !}{[(n / h)-(q / h) ! \times(q / h) !}\right\}^{h}$ possible solutions. According to the properties of combination number, there is $C_{n}^{q}=C_{n}^{n-q}$ when $q \leqslant \frac{n}{2}$ or $n-q \leqslant \frac{n}{2}$. In the case that $q / h$ is much smaller than $n / h$ (i.e., $q / h<<n / h), \Omega$ could be approximately processed as $\Omega=C_{n_{1}}^{q_{1}} \times C_{n_{2}}^{q_{2}} \times \cdots \times C_{n_{h}}^{q_{h}} \leqslant$ $\left[C_{n / h}^{q / h}\right]^{h}=\left\{\frac{(n / h) !}{[(n / h)-(q / h) ! \times(q / h) !}\right\}^{h} \leqslant\{(n / h) \times[(n / h)-1] \times \cdots \times[(n / h)-$ $(q / h)+1]\}^{h} \leqslant\left[(n / h)^{q / h}\right]^{h}=(n / h)^{q}$.

According to the above analysis, the solution space will approximately exponentially grow with increasing $q$. Since model (10)-(12) is an NP-hard problem, solving model (17)-(21) is more difficult than solving model (10)-(12). For the small-scale problem, i.e., $n / h$ and $q$ is very small, the traditional enumeration algorithm is capable. However, for the large-scale one, a meta-heuristic algorithm is required. Thus we develop an INSGA-II in the next section.

\section{Improved nondominated sorting genetic algorithm II (INSGA-II)}

Deb et al. (2002) proposed a nondominated sorting genetic algorithm II (NSGA-II). It is a kind of enlist and fast multi-objective genetic algorithm (MOGA). The NSGA-II significantly contributes to solving constrained multi-objective problems and it alleviates three difficulties in previous multi-objective evolutionary algorithms that use nondominated sorting and sharing, namely, computational complexity, nonelitism approach and the need for specifying a sharing parameter. In this section, an INSGA-II based on NSGA-II is developed to solve model (17)-(21). There are three reasons for developing the INSGA-II. Firstly, in the classical optimization methods, only one particular Pareto-optimal solution could be obtained in one time run, whereas genetic algorithm (GA) could simultaneously search a population of solutions in parallel. Therefore, we can obtain a solution set rather than only one solution using GA. Secondly, multi-objective genetic algorithm (MOGA) (see Deb et al., 2002) based on GA is quite suitable to solve NPHard multi-objective optimization problems. The existing research have shown that MOGA is effective in solving multi-objective problems in many fields (Mansouri, 2005; Tan et al., 2006; Lee et al., 2007, 2008; Jozefowiez et al., 2009). Additionally, we improve the NSGA-II to enhance its effectiveness and efficiency. In the following, according to the characteristics of model (17)-(21), the developed INSGA-II is described in detail.

\subsection{Representation and initialization}

Binary encoding is adopted to represent an individual as $[0,1$, $0, \ldots, 0,1]$ of $n$ codes (genes), where 1 denotes that the candidate is selected and 0 , otherwise. For model (17)-(21), there are total of $q$ genes encoded as 1 in each individual, which implies $q$ members need to be selected. The genes are divided into $h$ gene fragments ( $h$ is the total number of functional departments) and there are $q_{j}$ genes encoded as 1 in fragment $j$. The representation of an individual is shown in Fig. 3. According to binary encoding, individuals are randomly generated to form the initial population according to predetermined $n$ and $q$.

\subsection{Selection strategy}

The tournament selection is used, which starts with selecting $r$ individuals randomly, and then selecting the best one among them to survive in the next generation. A binary tournament $(r=2)$ is

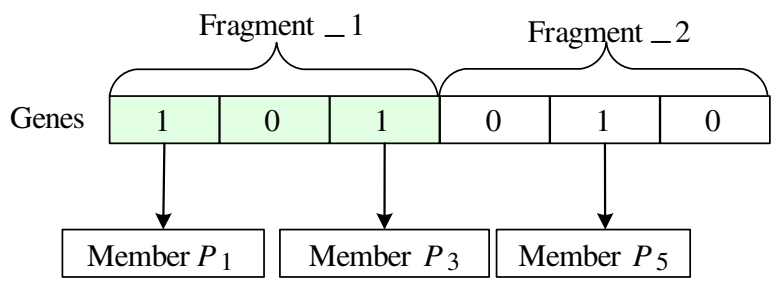

Fig. 3. The representation of an individual.

adopted. Obviously, such selection strategy guaranties the survival of best individuals during all generations. At the same time, it uses the relative fitness values as the standard for selection. Therefore, it can avoid the influence of super-individual, and avoid the occurrence of premature convergence and stagnation phenomenon to some extent.

\subsection{Crossover operator}

Two-point crossover is employed in this paper. At first, two crossover points are randomly selected in each fragment of the individual. Then the intervals between the two consecutive crossover points are exchanged (or swapped) between two parents to produce two new offsprings with crossover probability of $p_{c}$. However, this crossover operator may produce infeasible solutions (infeasible offsprings). For example, if $n=15, q=5, h=2, q_{1}=$ $3, q_{2}=2, S_{1}=\left\{P_{1}, P_{2}, P_{3}, P_{4}, P_{5}, P_{6}\right\} ; S_{2}=\left\{P_{7}, P_{8}, P_{9}, P_{10}, P_{11}, P_{12}, P_{13}\right.$, $\left.P_{14}, P_{15}\right\}$, then the two parents generated randomly are represented as:

parent_1 $=[1,0,1,0,1,0, \mid 0,0,1,0,0,0,0,0,1]$, parent_2 $=[0,1,1,0,0,1, \mid 1,0,0,0,1,0,0,0,0]$.

If the randomly generated crossover points are the 2 nd and 4 th points in gene fragment_1, then the two generated offsprings are represented as:

offspring_1 $=[1,1,1,0,1,0, \mid 0,0,1,0,0,0,0,0,1]$
offspring_2 $=[0,0,1,0,0,1, \mid 1,0,0,0,1,0,0,0,0]$.

It is obvious that the two offsprings in gene fragment_1 generated from crossover operator do not satisfy the constraint $q_{1}=3$, namely the solutions are infeasible. The existing research shows that reparation strategy for infeasible solutions is much better than the refusal strategy or penalty strategy in solving most combinational optimization problems (Orvosh and Davis, 1994). As such, the reparation strategy is designed as follows.

Randomly select one from the two generated offsprings and we assume that offspring_1 is selected:

- Let $q_{j}^{*}$ be total number of genes encoded as 1 in fragment_1 of offspring_1.

- If $q_{j}^{*}=q_{1}$, then the fragment_1 of offspring_1 is a feasible individual; otherwise, it should be repaired. If $q_{j}^{*}>q_{1}$, let $q_{j}^{*}-q_{1}$ genes be 0 , which are randomly selected from the genes encoded as 1 in fragment_1 of offspring_1. Meanwhile, let $q_{j}^{*}-q_{1}$ genes be 1 , which are randomly selected from the genes encoded as 0 in fragment_1 of offspring_2. On the contrary, if $q_{j}^{*}<q_{1}$, let $q_{j}-q_{j}^{*}$ genes be 1 , which are randomly selected from the genes encoded as 0 in fragment_1 of offspring_1 and let $q_{j}-q_{j}^{*}$ genes be 0 , which are randomly selected from the genes encoded as 1 in fragment_1 of offspring_2. The reparation ensures that the number of the genes encoded as 1 in fragment_ 1 is equal to $q_{1}$, i.e., $q_{j}^{*}=q_{1}$, and guarantees the individual feasibility. 
- Likewise, gene fragment_2 can be repaired by the above reparation strategy. This guarantees that there are $q_{j}(j=1,2, \ldots, h)$ genes encoded as 1 in fragment $j$.

\subsection{Mutation operator}

In this paper, the reverse mutation is used to each fragment of the individual. In this method, two-points in each fragment of the individual are randomly selected and the substring between them is reversed alternatively with mutation probability of $p_{m}$. Evidently, this method only changes the order of the genes but does not change the number of the genes encoded as 1 . Thus no infeasible solutions are produced.

\subsection{Fitness calculation}

In the single objective optimization model, the fitness function is usually the objective function. However, in the multi-objective models, the multi-objective functions should be reconsidered when calculating the fitness values. Based on the work of Deb et al. (2002), fast nondominated sorting approach (FNSA) is employed in INSGA-II. The fitness values of any two individuals are compared according to the dominated relationship and crowding distance in the objective space. Then all individuals are classified into the nondominated fronts. We note the first nondominated front as $F_{1}$, the second nondominated front as $F_{2}$ and so on. The worst case is that the number of fronts is equal to the population size (popSize) and only one solution in each front. The individuals in the latter fronts do not dominate those in the prior ones, and we call this nondominated sorting. At the same time, crowding distance is used to compare individuals. The small crowding distance of a individual indicates that there are many other individuals around it for the current population and the distribution of individuals is dense. As such, the individuals in different fronts are sorted again according to their crowding distances. In the same front, the individuals with larger crowding distance are more elitist. Additional details about FNSA can refer to Deb et al. (2002).

\subsection{Improvements of NSGA-II}

For MOGAs, including NSGA-II, they always face two difficulties (Deb, 1999). One is how to maintain population diversity to avoid premature convergence and make the solution have better distribution. The other is how to make choice so that the evolution of the population could lead to Pareto-optimal set as soon as possible. In order to alleviate the above difficulties and achieve better performance, we improved NSGA-II from two aspects.

First, a novel elite-preserving operator is proposed using a distribution function: $n f_{i}=\left|F_{i}\right| \cdot \xi^{\left(\frac{\max G e n}{g}\right)^{i}}$, where $n f_{i}$ denotes the num- ber of nondominated solutions that are selected from front $F_{i}$ to reproduce the new population $P_{(t+1)},\left|F_{i}\right|$ denotes the number of nondominated solutions in front $F_{i}$, and maxGen and $g$ are the numbers of maximum generation and current generation, respectively, $\xi, \lambda \in(0,1) ; i=1,2, \ldots, n$. This distribution function ensures more dominated solutions have the chances to survive in the earlier generations. Hence, the improved elite-preserving operator can avoid premature convergence and can maintain population diversity.

Second, the sorting and rejecting computation are improved. As for NSGA-II, in the generation $t$, the parent population (noted as $P_{t}$ ) and the offspring population (noted as $Q_{t}$ ) are combined together to form a new population (noted as $R_{t}$ ) of size $2 N$ (both the size of $P_{t}$ and $Q_{t}$ are $N$ ). Then, the individuals in population $R_{t}$ is classified to different nondominated fronts using FNSA. Since the popSize of $R_{t}$ is $2 N$, not all fronts can be accommodated with $N$ slots that are formed the new population $P_{(t+1)}$. Therefore, it is not necessary to classified all individuals in population $R_{t}$, and the classification should stop once the slots are filled by $N$ individuals. Such rejecting strategy is employed in the INSGA-II, and it shortens computational time and improves the algorithm efficiency. Fig. 4 vividly shows the difference between the procedure of NSGA-II and that of INSGA-II.

\section{Examples}

In this section, we present a real example on member selection of a CFT to illustrate the application of the proposed method. In addition, extensive computational experiments for comparing INSGA-II with NSGA-II are conducted to further test the effectiveness of INSGA-II.

SAC is one of the back-bone enterprises in the Aviation Industries of China. It has made great success in manufacturing, designing and developing aero-space vehicles, such as the double Mach number jet fighter aircraft, the in-flight fueling receiver jet fighter aircraft and so on. Simultaneously, SAC develops the non-aeroproducts by taking the advantage of aero-technology. It provides more than 100 kinds of products in seven ranges as military aircraft, automobile, light metal structures, large and medium-sized machinery equipment, electro-machinery and commercial airplane parts and components. With the globalization of economy, SAC is brought into the international business cooperation. Now, SAC cooperates with more than twenty international organizations for new product development and manufacture.

To conduct a radar development project, several CFTs need to be formed. The project will achieve the development of antenna and feeder subsystem, antenna pedestal subsystem, transmitter subsystem, receiver subsystem, velocity and range finding subsystem, signal and data processing subsystem, and monitored control subsystem. Taking the formation of CFT for developing antenna and

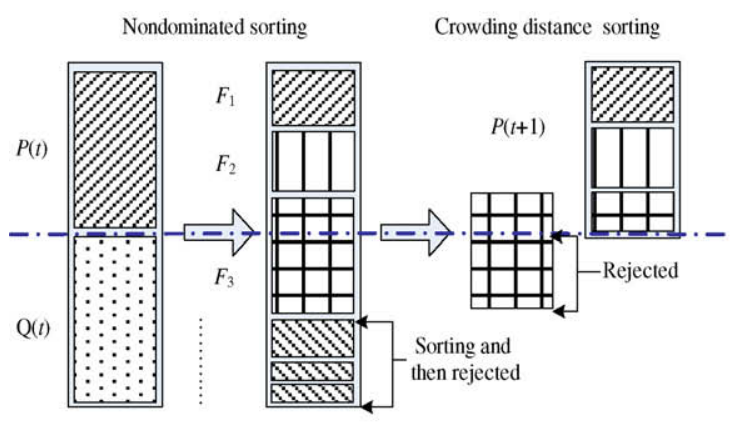

(a) NSGA-II procedure

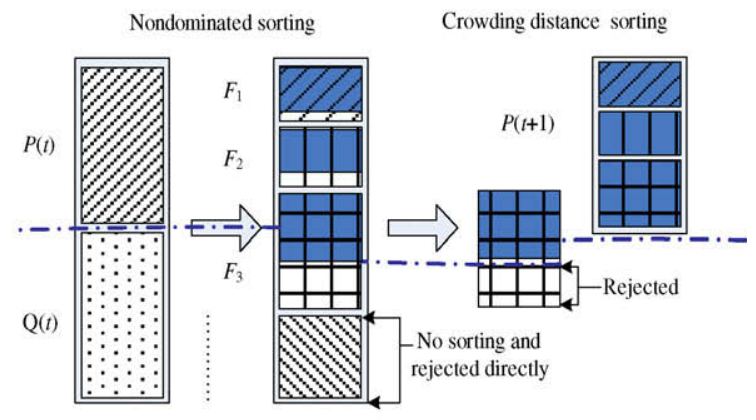

(b) INSGA-II procedure

Fig. 4. The procedures of NSGA-II and INSGA-II. 
Table 1

Criteria for member selection of the cross-functional team.

\begin{tabular}{|c|c|c|}
\hline Sub-objectives & Criteria & Descriptions \\
\hline \multirow[t]{3}{*}{ Individual performance } & Work experience $\left(I_{1}\right)$ & Years in the work domains \\
\hline & Work capability $\left(I_{2}\right)$ & Abilities to solve the problems in work \\
\hline & Specialized knowledge $\left(I_{3}\right)$ & The owned knowledge related to the task \\
\hline \multirow[t]{2}{*}{ Interior organizational collaborative performance } & Communication $\left(C_{1}^{(i n)}\right)$ & Mutual communication between members \\
\hline & Cooperation $\left(C_{2}^{(i n)}\right)$ & $\begin{array}{l}\text { Total number of problems that one helps another to } \\
\text { solve in the latest task }\end{array}$ \\
\hline Exterior organizational collaborative performance & Exterior organizational collaborative situation $\left(C^{(e x)}\right)$ & $\begin{array}{l}\text { The overall assessment on the exterior } \\
\text { organizational collaborative situation of candidate }\end{array}$ \\
\hline
\end{tabular}

feeder subsystem (AF team, thereafter) as an example, the requirements and organization process of $\mathrm{AF}$ team are presented as follows.

The AF team is composed of various types of positions and each type of position requires a developer of a specific discipline. Five desired members will be selected from twenty candidates of five disciplines, i.e., electronic engineering $\left(S_{1}\right)$, mechanical engineering $\left(S_{2}\right)$, mechanical process $\left(S_{3}\right)$, electronic process $\left(S_{4}\right)$, and standardization and quality management $\left(S_{5}\right)$. The number of the desired members from each discipline is $q_{1}=1, q_{2}=1, q_{3}=1, q_{4}=1$ and $q_{5}=1$. The candidate sets of the five disciplines are represented as $S_{1}=\left\{P_{1}, P_{2}, P_{3}, P_{4}, P_{5}, S_{2}=\left\{P_{6}, P_{7}, P_{8}\right\}, S_{3}=\left\{P_{9}, P_{10}\right\}, S_{4}=\left\{P_{11}\right.\right.$, $\left.P_{12}, P_{13}, P_{14}\right\}, S_{5}=\left\{P_{15}, P_{16}, P_{17}, P_{18}, P_{19}, P_{20}\right\}$, respectively.

The decision maker adopts six criteria for member selection (see Table 1 ). Herein, $I_{1}$ and $C_{2}^{(i n)}$ are objective criteria, while $I_{2}, I_{3}, C_{1}^{(i n)}$ and $C^{(e x)}$ are subjective ones. The data of criterion $I_{1}$ are obtained from the files of Department of Human Resource and

Table 2

The original data on criteria $I_{1}, I_{2}, I_{3}$ and $C_{1}^{(e x)}$.

\begin{tabular}{|c|c|c|c|c|c|c|c|c|c|}
\hline \multirow[t]{2}{*}{ Alternatives } & \multicolumn{4}{|c|}{ Criteria } & \multirow[t]{2}{*}{ Alternatives } & \multicolumn{4}{|c|}{ Criteria } \\
\hline & $I_{1}$ & $I_{2}$ & $I_{3}$ & $C^{(e x)}$ & & $I_{1}$ & $I_{2}$ & $I_{3}$ & $C^{(e x)}$ \\
\hline$P_{1}$ & 19 & 4 & 7 & 6 & $P_{11}$ & 12 & 7 & 7 & 5 \\
\hline$P_{2}$ & 10 & 6 & 2 & 7 & $P_{12}$ & 10 & 9 & 8 & 1 \\
\hline$P_{3}$ & 16 & 10 & 9 & 8 & $P_{13}$ & 7 & 5 & 7 & 5 \\
\hline$P_{4}$ & 21 & 9 & 8 & 9 & $P_{14}$ & 12 & 5 & 10 & 7 \\
\hline$P_{5}$ & 6 & 7 & 9 & 8 & $P_{15}$ & 23 & 8 & 4 & 8 \\
\hline$P_{6}$ & 15 & 7 & 8 & 9 & $P_{16}$ & 15 & 10 & 9 & 7 \\
\hline$P_{7}$ & 8 & 7 & 10 & 8 & $P_{17}$ & 9 & 9 & 7 & 9 \\
\hline$P_{8}$ & 12 & 8 & 4 & 8 & $P_{18}$ & 4 & 8 & 10 & 10 \\
\hline$P_{9}$ & 19 & 9 & 8 & 9 & $P_{19}$ & 10 & 10 & 7 & 8 \\
\hline$P_{10}$ & 5 & 2 & 7 & 5 & $P_{20}$ & 16 & 9 & 5 & 10 \\
\hline
\end{tabular}

Table 3

The original data on criterion $C_{1}^{(i n)}$.

\begin{tabular}{|c|c|c|c|c|c|c|c|c|c|c|c|c|c|c|c|c|c|c|c|c|}
\hline & $P_{1}$ & $P_{2}$ & $P_{3}$ & $P_{4}$ & $P_{5}$ & $P_{6}$ & $P_{7}$ & $P_{8}$ & $P_{9}$ & $P_{10}$ & $P_{11}$ & $P_{12}$ & $P_{13}$ & $P_{14}$ & $P_{15}$ & $P_{16}$ & $P_{17}$ & $P_{18}$ & $P_{19}$ & $P_{20}$ \\
\hline$P_{1}$ & - & 7 & 8 & 5 & 6 & 3 & 1 & 4 & 6 & 5 & 9 & 7 & 6 & 6 & 3 & 2 & 7 & 1 & 2 & 8 \\
\hline$P_{2}$ & 7 & - & 7 & 9 & 2 & 1 & 9 & 4 & 4 & 1 & 5 & 8 & 8 & 7 & 7 & 2 & 1 & 9 & 6 & 5 \\
\hline$P_{3}$ & 8 & 7 & - & 10 & 1 & 8 & 9 & 9 & 9 & 7 & 7 & 8 & 8 & 4 & 2 & 8 & 3 & 8 & 9 & 2 \\
\hline$P_{4}$ & 5 & 9 & 10 & - & 3 & 3 & 8 & 2 & 10 & 1 & 9 & 8 & 8 & 7 & 7 & 9 & 1 & 8 & 6 & 7 \\
\hline$P_{5}$ & 6 & 2 & 1 & 3 & - & 2 & 6 & 8 & 7 & 4 & 8 & 2 & 4 & 5 & 3 & 8 & 3 & 5 & 4 & 6 \\
\hline$P_{6}$ & 3 & 1 & 8 & 3 & 2 & - & 1 & 7 & 3 & 4 & 8 & 5 & 1 & 8 & 6 & 10 & 4 & 8 & 2 & 4 \\
\hline$P_{7}$ & 1 & 9 & 9 & 8 & 6 & 1 & - & 5 & 8 & 3 & 8 & 8 & 1 & 4 & 3 & 9 & 1 & 6 & 8 & 5 \\
\hline$P_{8}$ & 4 & 4 & 9 & 2 & 8 & 7 & 5 & - & 6 & 4 & 6 & 3 & 7 & 2 & 1 & 5 & 3 & 8 & 2 & 4 \\
\hline$P_{9}$ & 6 & 4 & 9 & 10 & 7 & 3 & 8 & 6 & - & 6 & 8 & 9 & 5 & 6 & 5 & 10 & 1 & 5 & 8 & 7 \\
\hline$P_{10}$ & 5 & 1 & 7 & 1 & 4 & 4 & 3 & 4 & 6 & - & 6 & 7 & 2 & 3 & 4 & 8 & 5 & 5 & 7 & 5 \\
\hline$P_{11}$ & 9 & 5 & 7 & 9 & 8 & 8 & 8 & 6 & 8 & 6 & - & 6 & 6 & 8 & 5 & 6 & 6 & 8 & 5 & 3 \\
\hline$P_{12}$ & 7 & 8 & 8 & 8 & 2 & 5 & 8 & 3 & 9 & 7 & 6 & - & 4 & 6 & 2 & 8 & 7 & 8 & 7 & 2 \\
\hline$P_{13}$ & 6 & 8 & 8 & 8 & 4 & 1 & 1 & 7 & 5 & 2 & 6 & 4 & - & 6 & 2 & 8 & 4 & 6 & 6 & 5 \\
\hline$P_{14}$ & 6 & 7 & 4 & 7 & 5 & 8 & 4 & 2 & 6 & 3 & 8 & 6 & 6 & - & 6 & 7 & 3 & 6 & 6 & 8 \\
\hline$P_{15}$ & 3 & 7 & 2 & 7 & 3 & 6 & 3 & 1 & 5 & 4 & 5 & 2 & 2 & 6 & - & 6 & 4 & 7 & 3 & 5 \\
\hline$P_{16}$ & 2 & 2 & 8 & 9 & 8 & 10 & 9 & 5 & 10 & 8 & 6 & 8 & 8 & 7 & 6 & - & 4 & 6 & 10 & 8 \\
\hline$P_{17}$ & 7 & 1 & 3 & 1 & 3 & 4 & 1 & 3 & 1 & 5 & 6 & 7 & 4 & 3 & 4 & 4 & - & 3 & 8 & 1 \\
\hline$P_{18}$ & 1 & 9 & 8 & 8 & 5 & 8 & 6 & 8 & 5 & 5 & 8 & 8 & 6 & 6 & 7 & 6 & 3 & - & 4 & 3 \\
\hline$P_{19}$ & 2 & 6 & 9 & 6 & 4 & 2 & 8 & 2 & 8 & 7 & 5 & 7 & 6 & 6 & 3 & 10 & 8 & 4 & - & 2 \\
\hline$P_{20}$ & 8 & 5 & 2 & 7 & 6 & 4 & 5 & 4 & 7 & 5 & 3 & 2 & 5 & 8 & 5 & 8 & 1 & 3 & 2 & - \\
\hline
\end{tabular}

the data of criterion $C_{2}^{(i n)}$ is obtained from the reports of Collaborative System. The values of criteria $I_{2}, I_{3}, C_{1}^{(i n)}$ and $C^{(e x)}$ are provided by the decision maker using the scores from 1 to 10 (1: very bad, 10: very good). The original data is shown in Tables $2-4$.

The original data with regard to criteria $I_{1}, I_{2}$ and $I_{3}$ is normalized by Eq. (1). Likewise, the data with regard to criteria $C_{1}^{(i n)}$ and $C_{2}^{(i n)}$ is normalized by Eq. (7). The decision maker finalizes criterion weights by direct assignment. That is, the weights of criteria $I_{1}, I_{2}$ and $I_{3}$ are $v_{1}=0.3, v_{2}=0.4$ and $v_{3}=0.3$, respectively, and the weights of criteria of $C_{1}^{(i n)}$ and $C_{2}^{(i n)}$ are $w_{1}=0.6, w_{2}=0.4$, respectively. Furthermore, the overall values of individual performance and collaborative performance are presented in Table 5. In Table 5 , the overall values of individual performance $\left(\varphi_{i}, i=1\right.$, $2, \ldots, 20)$ are obtained by Eq. (3). The overall values of interior organizational performance $\left(\phi_{i j}^{(i n)}, i, j=1,2, \ldots, 20, i \neq j\right)$ are obtained by Eq. (9). The normalized assessment values of exterior organizational performance $\left(a_{i}^{\prime(e x)}, i=1,2, \ldots, 20\right)$ are obtained by Eq. (13).

Based on model (17)-(21), the model for member selections of the real example can be built as follows:

$$
\begin{array}{cc}
\text { Maximize } & Z_{1}=0.52 x_{1}+0.29 x_{2}+0.85 x_{3}+\cdots+0.68 x_{19}+0.65 x_{20}, \\
\text { Maximize } & Z_{2}=0.61 x_{1} x_{2}+0.63 x_{1} x_{3}+0.46 x_{1} x_{4} \\
& +\cdots+0.17 x_{20} x_{18}+0.15 x_{20} x_{19}, \\
\text { Maximize } \quad & Z_{3}=0.56 x_{1}+0.67 x_{2}+0.78 x_{3}+\cdots+x_{18}+0.78 x_{19}+x_{20}, \\
\text { subject to } & \sum_{i=1}^{5} x_{i}=1, \quad \sum_{i=6}^{8} x_{i}=1, \sum_{i=9}^{10} x_{i}=1, \quad \sum_{i=11}^{14} x_{i}=1 \sum_{i=15}^{20} x_{i}=1, \\
& x_{i}=1 \text { or } 0, \quad i=1,2, \ldots, 20 .
\end{array}
$$

To solve the above problem, INSGA-II developed in Section 4 is employed. The parameters are set as popSize $=30, \operatorname{maxGen}=20$, 
Table 4

The original data on criterion $C_{2}^{(i n)}$.

\begin{tabular}{|c|c|c|c|c|c|c|c|c|c|c|c|c|c|c|c|c|c|c|c|c|}
\hline & $P_{1}$ & $P_{2}$ & $P_{3}$ & $P_{4}$ & $P_{5}$ & $P_{6}$ & $P_{7}$ & $P_{8}$ & $P_{9}$ & $P_{10}$ & $P_{11}$ & $P_{12}$ & $P_{13}$ & $P_{14}$ & $P_{15}$ & $P_{16}$ & $P_{17}$ & $P_{18}$ & $P_{19}$ & $P_{20}$ \\
\hline$P_{1}$ & - & 13 & 10 & 12 & 6 & 8 & 6 & 9 & 3 & 5 & 13 & 14 & 7 & 9 & 6 & 15 & 5 & 7 & 11 & 15 \\
\hline$P_{2}$ & 7 & - & 14 & 15 & 5 & 5 & 9 & 0 & 5 & 6 & 0 & 12 & 0 & 4 & 0 & 11 & 15 & 8 & 15 & 5 \\
\hline$P_{3}$ & 11 & 4 & - & 23 & 3 & 2 & 19 & 11 & 22 & 5 & 13 & 19 & 15 & 12 & 11 & 12 & 9 & 13 & 16 & 11 \\
\hline$P_{4}$ & 12 & 17 & 15 & - & 8 & 13 & 12 & 5 & 20 & 13 & 0 & 18 & 0 & 6 & 9 & 16 & 15 & 11 & 19 & 5 \\
\hline$P_{5}$ & 7 & 0 & 8 & 19 & - & 9 & 2 & 12 & 3 & 1 & 12 & 4 & 4 & 13 & 15 & 0 & 7 & 26 & 5 & 8 \\
\hline$P_{6}$ & 5 & 5 & 3 & 15 & 14 & - & 9 & 13 & 10 & 10 & 13 & 8 & 6 & 5 & 11 & 6 & 3 & 7 & 4 & 3 \\
\hline$P_{7}$ & 8 & 4 & 22 & 19 & 3 & 11 & - & 8 & 16 & 25 & 8 & 21 & 6 & 5 & 5 & 20 & 9 & 6 & 25 & 14 \\
\hline$P_{8}$ & 4 & 0 & 9 & 8 & 7 & 9 & 13 & - & 11 & 8 & 7 & 5 & 0 & 8 & 6 & 3 & 3 & 8 & 15 & 8 \\
\hline$P_{9}$ & 9 & 11 & 23 & 16 & 1 & 13 & 18 & 7 & - & 13 & 11 & 17 & 13 & 2 & 9 & 20 & 25 & 0 & 22 & 6 \\
\hline$P_{10}$ & 2 & 0 & 7 & 2 & 2 & 3 & 11 & 3 & 3 & - & 5 & 9 & 12 & 12 & 11 & 24 & 6 & 15 & 0 & 10 \\
\hline$P_{11}$ & 4 & 0 & 11 & 13 & 14 & 6 & 4 & 12 & 9 & 4 & - & 5 & 14 & 18 & 14 & 0 & 5 & 10 & 12 & 11 \\
\hline$P_{12}$ & 13 & 3 & 13 & 22 & 25 & 3 & 26 & 14 & 20 & 5 & 11 & - & 3 & 5 & 7 & 25 & 3 & 0 & 18 & 14 \\
\hline$P_{13}$ & 10 & 2 & 5 & 9 & 0 & 0 & 10 & 9 & 4 & 3 & 3 & 13 & - & 4 & 4 & 9 & 4 & 0 & 7 & 3 \\
\hline$P_{14}$ & 6 & 5 & 0 & 11 & 1 & 17 & 8 & 6 & 5 & 10 & 11 & 15 & 19 & - & 15 & 0 & 4 & 13 & 4 & 14 \\
\hline$P_{15}$ & 9 & 11 & 10 & 11 & 15 & 9 & 5 & 0 & 0 & 11 & 7 & 6 & 9 & 12 & - & 9 & 7 & 0 & 5 & 10 \\
\hline$P_{16}$ & 17 & 6 & 15 & 18 & 3 & 9 & 21 & 14 & 18 & 12 & 15 & 24 & 7 & 14 & 10 & - & 12 & 6 & 18 & 12 \\
\hline$P_{17}$ & 8 & 2 & 10 & 11 & 13 & 13 & 9 & 0 & 11 & 10 & 9 & 13 & 6 & 3 & 9 & 0 & - & 6 & 14 & 8 \\
\hline$P_{18}$ & 13 & 6 & 13 & 2 & 6 & 4 & 6 & 5 & 4 & 10 & 3 & 8 & 5 & 6 & 13 & 9 & 5 & - & 6 & 11 \\
\hline$P_{19}$ & 6 & 15 & 18 & 20 & 5 & 6 & 11 & 14 & 19 & 9 & 10 & 15 & 15 & 9 & 11 & 18 & 6 & 2 & - & 3 \\
\hline$P_{20}$ & 13 & 9 & 14 & 10 & 0 & 11 & 9 & 0 & 6 & 6 & 13 & 8 & 2 & 4 & 8 & 7 & 11 & 2 & 5 & - \\
\hline
\end{tabular}

Table 5

The overall values of the individual and collaborative performances.

\begin{tabular}{|c|c|c|c|c|c|c|c|c|c|c|c|c|c|c|c|c|c|c|c|c|c|}
\hline & & $P_{1}$ & $P_{2}$ & $P_{3}$ & $P_{4}$ & $P_{5}$ & $P_{6}$ & $P_{7}$ & $P_{8}$ & $P_{9}$ & $P_{10}$ & $P_{11}$ & $P_{12}$ & $P_{13}$ & $P_{14}$ & $P_{15}$ & $P_{16}$ & $P_{17}$ & $P_{18}$ & $P_{19}$ & $P_{20}$ \\
\hline \multirow{20}{*}{$\phi_{i j}^{(i n)}$} & $P_{1}$ & - & .61 & .63 & .46 & .43 & .26 & .10 & .34 & .38 & .35 & .74 & .62 & .45 & .48 & .23 & .31 & .48 & .11 & .24 & .71 \\
\hline & $P_{2}$ & .51 & - & .62 & .77 & .15 & .08 & .68 & .20 & .28 & .10 & .27 & .66 & .47 & .46 & .40 & .24 & .24 & .66 & .57 & .35 \\
\hline & $P_{3}$ & .64 & .46 & - & .97 & .05 & .50 & .84 & .71 & .89 & .48 & .61 & .77 & .71 & .39 & .24 & .66 & .28 & .67 & .79 & .24 \\
\hline & $P_{4}$ & .46 & .81 & .84 & - & .26 & .34 & .66 & .15 & .92 & .21 & .53 & .75 & .47 & .50 & .54 & .79 & .24 & .64 & .64 & .48 \\
\hline & $P_{5}$ & .45 & .07 & .13 & .44 & - & .21 & .37 & .66 & .45 & .22 & .66 & .13 & .26 & .47 & .37 & .47 & .25 & .68 & .28 & .46 \\
\hline & $P_{6}$ & .21 & .08 & .51 & .37 & .29 & - & .14 & .61 & .29 & .36 & .67 & .39 & .10 & .55 & .51 & .70 & .25 & .58 & .13 & .25 \\
\hline & $P_{7}$ & .13 & .60 & .89 & .77 & .38 & .18 & - & .39 & .72 & .53 & .59 & .80 & .10 & .28 & .21 & .85 & .14 & .43 & .87 & .49 \\
\hline & $P_{8}$ & .26 & .20 & .68 & .19 & .58 & .54 & .47 & - & .51 & .33 & .45 & .21 & .40 & .19 & .10 & .31 & .18 & .59 & .31 & .33 \\
\hline & $P_{9}$ & .48 & .38 & .90 & .86 & .42 & .34 & .75 & .45 & - & .54 & .64 & .81 & .47 & .37 & .41 & .92 & .40 & .27 & .82 & .50 \\
\hline & $P_{10}$ & .30 & 0 & .51 & .03 & .23 & .25 & .31 & .25 & .38 & - & .41 & .54 & .26 & .33 & .38 & .85 & .36 & .51 & .40 & .43 \\
\hline & $P_{11}$ & .60 & .27 & .58 & .74 & .69 & .56 & .53 & .53 & .61 & .40 & - & .41 & .56 & .75 & .49 & .33 & .41 & .63 & .46 & .31 \\
\hline & $P_{12}$ & .61 & .51 & .67 & .82 & .47 & .31 & .88 & .36 & .85 & .48 & .51 & - & .25 & .41 & .18 & .87 & .45 & .47 & .69 & .29 \\
\hline & $P_{13}$ & .49 & .50 & .55 & .61 & .20 & 0 & .16 & .54 & .33 & .11 & .38 & .41 & - & .40 & .13 & .61 & .26 & .33 & .45 & .31 \\
\hline & $P_{14}$ & .43 & .48 & .20 & .58 & .28 & .74 & .33 & .16 & .41 & .29 & .64 & .57 & .64 & - & .57 & .40 & .20 & .54 & .40 & .69 \\
\hline & $P_{15}$ & .28 & .58 & .23 & .58 & .37 & .48 & .21 & 0 & .27 & .38 & .38 & .16 & .21 & .53 & - & .48 & .31 & .40 & .21 & .43 \\
\hline & $P_{16}$ & .34 & .16 & .71 & .82 & .51 & .74 & .87 & .49 & .89 & .66 & .57 & .85 & .58 & .62 & .49 & - & .39 & .43 & .89 & .66 \\
\hline & $P_{17}$ & .53 & .03 & .29 & .18 & .34 & .41 & .14 & .13 & .18 & .43 & .48 & .61 & .30 & .18 & .34 & .20 & - & .23 & .69 & .13 \\
\hline & $P_{18}$ & .21 & .63 & .67 & .50 & .36 & .53 & .43 & .55 & .33 & .43 & .51 & .59 & .41 & .43 & .61 & .48 & .21 & - & .30 & .31 \\
\hline & $P_{19}$ & .16 & .57 & .82 & .65 & .28 & .16 & .64 & .29 & .77 & .54 & .43 & .64 & .57 & .48 & .31 & .89 & .56 & .23 & - & .11 \\
\hline & $P_{20}$ & .67 & .41 & .29 & .56 & .33 & .38 & .41 & .20 & .50 & .36 & .34 & .19 & .30 & .53 & .39 & .58 & .18 & .17 & .15 & - \\
\hline$\varphi_{i}$ & & .52 & .29 & .85 & .84 & .54 & .65 & .61 & .50 & .81 & .20 & .56 & .67 & .38 & .58 & .68 & .84 & .62 & .60 & .68 & .65 \\
\hline$a_{i}^{\prime(e x)}$ & & .56 & .67 & .78 & .89 & .78 & .89 & .78 & .78 & .89 & .44 & .44 & .0 & .44 & .67 & .78 & .67 & .89 & 1 & .78 & .1 \\
\hline
\end{tabular}

$p_{c}=0.95, p_{m}=0.05, \xi=0.98$ and $\lambda=0.5$. INSGA-II is coded in VB.NET and run on a PC with an Intel Core2 $2.8 \mathrm{GHz}$ CPU and $1 \mathrm{GBy}-$ tes RAM.

INSGA-II is implemented ten times, and the average runtime is 0.0266 second. The obtained Pareto-optimal solutions are listed in Table 6. On the other hand, enumeration algorithm is also introduced to solve the above problem (the average runtime is 5.0734 second) and all the feasible solutions are presented in Fig. 5. The above comparison results demonstrates that INSGA-II is much faster than enumeration algorithm and can obtain all true Paretooptimal solutions (see Table 6 and Fig. 5).

To further test the performance of INSGA-II, extensive computational experiments are carried out (see Table 7). The developed algorithm performs after extensive testing experiments with changing parameters. The finalized parameters are shown in Table 7. In the eight large-scale examples, the overall values of individual performance and collaborative performance are generated with random numbers from the standard uniform distribution $N(0,1)$.
Usually, it is intractable to solve the large-scale NP-hard problems using enumeration algorithm. Thus we only compare INSGA-II with NSGA-II (Deb et al., 2002) by solving the above eight large-scale experimental examples. Referring to the work of Deb et al. (2002), three metrics, $\Upsilon, \Delta$ and $t$, are employed to make a comprehensive and quantitative comparison between INSGA-II and NSGA-II. Herein $\Upsilon$ measures the extent of convergence to a reference set. This reference set (also named pseudo-optimal Pareto front) is the integration of nondominated solutions obtained by the two algorithms; $\Delta$ measures the extent of spread achieved among the obtained solutions; $t$ measures the runtime of the algorithm.

Since both INSGA-II and NSGA-II belong to stochastic search algorithms with a stochastic nature, the nondominated (Paretooptimal or approximated Pareto-optimal) solutions may change in different runs. For this reason, two algorithms have been run ten times for each example and the computational results are listed in Table 8. 
Table 6

The computational results of the real example.

\begin{tabular}{llll} 
Pareto-optimal solutions (objective space) & \multicolumn{2}{l}{ Optimal objective } \\
$X^{*}=\left(x_{1}^{*}, x_{2}^{*}, x_{3}^{*}, \ldots, x_{19}^{*}, x_{20}^{*}\right)^{T}$ & \multicolumn{2}{l}{ function values } \\
\cline { 2 - 4 }$(0,0,0,1,0,0,1,0,1,0,0,0,0,1,0,0,0,0,0,1)^{T}$ & 3.49 & 11.31 & 4.23 \\
$(0,0,0,1,0,0,1,0,1,0,1,0,0,0,0,0,0,0,1,0)^{T}$ & 3.5 & 13.6 & 3.78 \\
$(0,0,1,0,0,0,1,0,1,0,0,0,0,1,0,0,0,0,0,1)^{T}$ & 3.5 & 10.62 & 4.12 \\
$(0,0,0,1,0,0,1,0,1,0,0,0,0,1,0,0,0,0,1,0)^{T}$ & 3.52 & 12.42 & 4.01 \\
$(0,0,1,0,0,0,1,0,1,0,0,0,0,1,0,0,0,0,1,0)^{T}$ & 3.53 & 12.56 & 3.9 \\
$(0,0,0,1,0,1,0,0,1,0,0,0,0,1,0,0,0,0,0,1)^{T}$ & 3.53 & 10.16 & 4.34 \\
$(0,0,1,0,0,1,0,0,1,0,0,0,0,1,0,0,0,0,0,1)^{T}$ & 3.54 & 9.47 & 4.23 \\
$(0,0,0,1,0,1,0,0,1,0,0,0,0,1,0,0,0,0,1,0)^{T}$ & 3.56 & 10.32 & 4.12 \\
$(0,0,0,1,0,0,1,0,1,0,0,1,0,0,0,0,0,0,1,0)^{T}$ & 3.61 & 15.31 & 3.34 \\
$(0,0,1,0,0,0,1,0,1,0,0,1,0,0,0,0,0,0,1,0)^{T}$ & 3.62 & 15.81 & 3.23 \\
$(0,0,0,1,0,0,1,0,1,0,1,0,0,0,0,1,0,0,0,0)^{T}$ & 3.66 & 14.36 & 3.67 \\
$(0,0,1,0,0,0,1,0,1,0,1,0,0,0,0,1,0,0,0,0)^{T}$ & 3.67 & 14.35 & 3.56 \\
$(0,0,0,1,0,0,1,0,1,0,0,0,0,1,0,1,0,0,0,0)^{T}$ & 3.68 & 13.31 & 3.9 \\
$(0,0,1,0,0,0,1,0,1,0,0,0,0,1,0,1,0,0,0,0)^{T}$ & 3.69 & 12.89 & 3.79 \\
$(0,0,0,1,0,1,0,0,1,0,1,0,0,0,0,1,0,0,0,0)^{T}$ & 3.7 & 12.63 & 3.78 \\
$(0,0,1,0,0,1,0,0,1,0,1,0,0,0,0,1,0,0,0,0)^{T}$ & 3.71 & 12.62 & 3.67 \\
$(0,0,0,1,0,1,0,0,1,0,0,0,0,1,0,1,0,0,0,0)^{T}$ & 3.72 & 12.15 & 4.01 \\
$(0,0,1,0,0,1,0,0,1,0,0,0,0,1,0,1,0,0,0,0)^{T}$ & 3.73 & 11.73 & 3.9 \\
$(0,0,0,1,0,0,1,0,1,0,0,1,0,0,0,1,0,0,0,0)^{T}$ & 3.77 & 16.45 & 3.23 \\
$(0,0,1,0,0,0,1,0,1,0,0,1,0,0,0,1,0,0,0,0)^{T}$ & 3.78 & 16.39 & 3.12 \\
$(0,0,0,1,0,1,0,0,1,0,0,1,0,0,0,1,0,0,0,0)^{T}$ & 3.81 & 13.63 & 3.34 \\
$(0,0,1,0,0,1,0,0,1,0,0,1,0,0,0,1,0,0,0,0)^{T}$ & 3.82 & 13.57 & 3.23 \\
& & & \\
& & &
\end{tabular}

It can be seen from Table 8 that the mean $\bar{\Upsilon}$ obtained by INSGAII is smaller than those obtained by NSGA-II in all examples. The variance $\sigma_{\Upsilon}$ obtained by INSGA-II is smaller than those obtained by NSGA-II except for Nos. 7 and 8. The comparison results show that nondominated solutions obtained by INSGA-II are closer to the pseudo-optimal Pareto front than those obtained by NSGA-II. Thus INSGA-II would converge better in most of cases. Furthermore, the mean $\bar{\Delta}$ and variance $\sigma_{\Delta}$ indicate that INSGA-II has a wider spread of solutions than NSGA-II in most cases. Moreover, INSGA-II is slightly faster than NSGA-II in all cases which can be seen from the mean $\bar{t}$. The main reason is that the sorting and rejecting computation adopted in INSGA-II could save more runtime. In sum, compared with NSGA-II, INSGA-II can produce a bet-

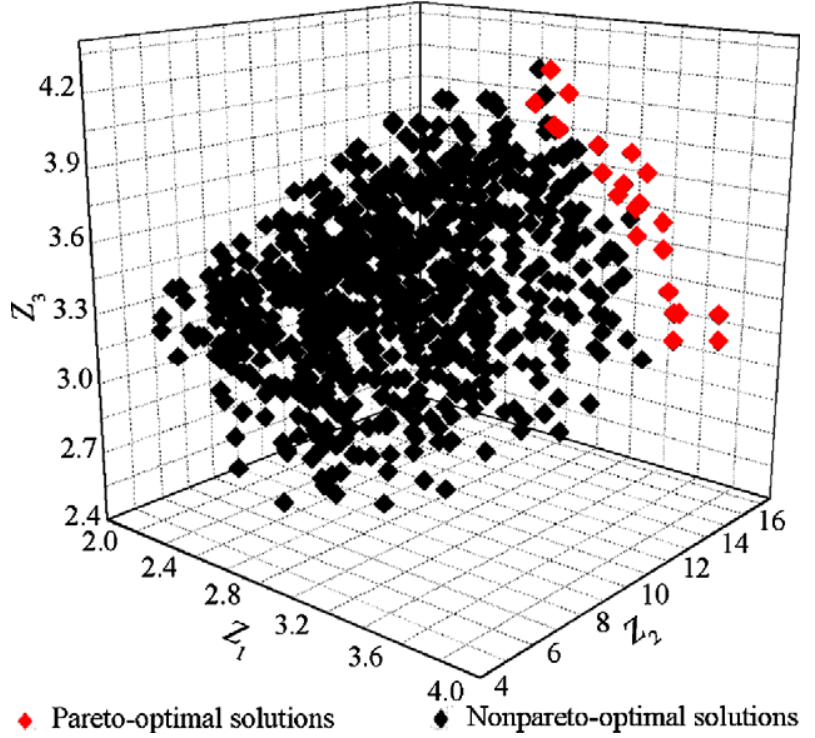

Fig. 5. The all solutions of the real example.

ter convergence and distribution of nondominated solutions with less runtime in most of large-scale examples. The above results and analyses indicate that INSGA-II can be used to solve the current model and more suitable than NSGA-II.

\section{Conclusions}

Not only the individual performance of candidates but also the collaborative performance between candidates is important for member selection in the formation of CFTs. To overcome the limitation of less consideration of collaborative performance in the existing methods, this paper presents a method for solving such a problem using both individual performance and collaborative performance. The major contributions of the proposed method are discussed bellow.

Table 7

Eight large-scale experimental examples.

\begin{tabular}{|c|c|c|c|c|c|c|c|c|c|c|c|}
\hline No. & $n$ & $q$ & {$\left[n_{1, \ldots} n_{5}\right]$} & {$\left[q_{1, \ldots} q_{5}\right]$} & popSize & maxGen & $p_{c}$ & $p_{m}$ & $\xi$ & $\lambda$ & Solution space $\Omega$ \\
\hline 1 & 50 & 5 & {$[10,10,10,10,10]$} & {$[1,1,1,1,1]$} & 60 & 100 & 0.95 & 0.05 & 0.98 & 0.5 & $O\left(10^{5}\right)$ \\
\hline 2 & 100 & 5 & {$[20,20,20,20,20]$} & {$[1,1,1,1,1]$} & 80 & 200 & 0.95 & 0.05 & 0.98 & 0.5 & $O\left(20^{5}\right)$ \\
\hline 3 & 150 & 5 & {$[30,30,30,30,30]$} & {$[1,1,1,1,1]$} & 100 & 200 & 0.95 & 0.05 & 0.98 & 0.5 & $O\left(30^{5}\right)$ \\
\hline 4 & 150 & 10 & {$[30,30,30,30,30]$} & {$[2,2,2,2,2]$} & 100 & 200 & 0.95 & 0.05 & 0.98 & 0.5 & $O\left(30^{10}\right)$ \\
\hline 5 & 150 & 15 & {$[30,30,30,30,30]$} & {$[3,3,3,3,3]$} & 100 & 200 & 0.95 & 0.05 & 0.98 & 0.5 & $O\left(30^{15}\right)$ \\
\hline 6 & 200 & 15 & {$[40,40,40,40,40]$} & {$[3,3,3,3,3]$} & 100 & 200 & 0.95 & 0.05 & 0.98 & 0.5 & $O\left(40^{15}\right)$ \\
\hline 7 & 300 & 20 & {$[60,60,60,60,60]$} & {$[4,4,4,4,4]$} & 120 & 200 & 0.95 & 0.05 & 0.98 & 0.5 & $O\left(60^{20}\right)$ \\
\hline 8 & 400 & 30 & {$[80,80,80,80,80]$} & {$[6,6,6,6,6]$} & 140 & 200 & 0.95 & 0.05 & 0.98 & 0.5 & $O\left(80^{30}\right)$ \\
\hline
\end{tabular}

Table 8

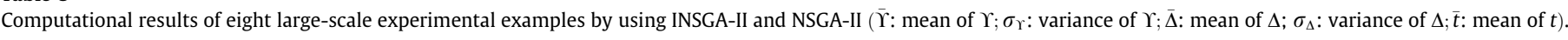

\begin{tabular}{|c|c|c|c|c|c|c|c|c|c|c|}
\hline \multirow[t]{2}{*}{ No. } & \multicolumn{5}{|l|}{ INSGA-II } & \multicolumn{5}{|l|}{ NSGA-II } \\
\hline & $\bar{\Upsilon}$ & $\sigma_{\Upsilon}$ & $\bar{\Delta}$ & $\sigma_{\Delta}$ & $\bar{t}(\mathrm{~s})$ & $\bar{\Upsilon}$ & $\sigma_{\Upsilon}$ & $\bar{\Delta}$ & $\sigma_{\Delta}$ & $\bar{t}(\mathrm{~s})$ \\
\hline 1 & .080371 & .002285 & .605040 & .009311 & 0.4469 & .089526 & .006038 & .620702 & .011140 & 0.4531 \\
\hline 2 & .065924 & .000818 & .592774 & .002165 & 1.7609 & .067671 & .000983 & .578735 & .004745 & 1.7656 \\
\hline 3 & .097788 & .001242 & .572687 & .003777 & 2.7875 & .117005 & .002118 & .599070 & .002194 & 2.8266 \\
\hline 4 & .050646 & .000168 & .619965 & .002975 & 2.7828 & .064753 & .000814 & .621089 & .005879 & 2.8188 \\
\hline 5 & .050682 & .000415 & .682124 & .006523 & 2.7703 & .060240 & .001024 & .684600 & .006882 & 2.7891 \\
\hline 6 & .058187 & .000477 & .704375 & .015610 & 3.0266 & .069279 & .000604 & .751813 & .010856 & 3.0469 \\
\hline 7 & .039982 & .000510 & .728559 & .005816 & 4.7375 & .051218 & .000208 & .794478 & .007839 & 4.7579 \\
\hline 8 & .038256 & .000294 & .719771 & .003183 & 6.8641 & .042470 & .000081 & .757823 & .012481 & 6.9547 \\
\hline
\end{tabular}


First, it is a new idea to consider the individual performance of candidates and the collaborative performance between candidates together for member selection in the formation of CFTs. It overcomes the limitations that occur in the existing methods considering either the individual performance or the collaborative performance. It also helps to reduce the uncertainty regarding the cooperation among the potential members.

Second, a multi-objective $0-1$ programming model is built to select desired members from different functional departments. In the model, individual performance, interior organizational collaborative performance and exterior organizational collaborative performance are considered. It provides a basic model for further research of related issues. The model can be also embedded in the decision support system to process more complex decision problem of member selection of CFTs using the individual and collaborative performances.

Third, an INSGA-II is developed to solve the model. It improved the elite-preserving operator and the sorting and rejecting computation of NSGA-II. The real example along with extensive computational experiments shows the effectiveness and efficiency of the proposed algorithm. Compared with NSGA-II, INSGA-II can produce a better convergence and distribution of nondominated solutions with less runtime in most of large-scale examples.

Additionally, a real example of CFT formation for radar development in SAC Company is presented to illustrate the application of the method proposed in this paper.

In terms of future research, the application of the proposed method can be extended to specific backgrounds such as concurrent engineering teams, new product development teams, originality teams and so on. It needs to be noted that, due to the limited resources, diversified requirements of team formation and different preferences of the decision maker, the proposed model can be modified by adding goals or constraints before it is applied to solve other problems. Moreover, we intend to develop a decision support system, in which the proposed model and algorithm are embedded. The decision support system will be universal and convenient to support decision makers.

\section{Acknowledgements}

This work was partly supported by the National Natural Science Funds for Distinguished Young Scientists of China (Project No. 70525002), National Natural Science Funds for Creative Research Groups of China (Project No. 70721001), National Natural Science Funds of China (Project Nos. 70901027 and 70801012) and China Postdoctoral Science Funds (Project No. 20080441087). Gratitude is also extended to Editor Roman Slowinski and the anonymous reviewers for their valuable comments.

\section{References}

Ancona, D.G., 1990. Outward bound: Strategies for team survival in an organization. Academy of Management Journal 33, 334-365.

Balakrishnan, S., Koza, M.P., 1993. Information asymmetry, adverse selection and joint ventures. Journal of Economic Behavior and Organization 20, 99-117.

Campion, M.A., Medsker, G.J., Higgs, A.C., 1993. Relations between work group characteristics and effectiveness: Implications for designing effective work groups. Personal Psychology 46, 823-850.

Chen, S.J., Lin, L., 2004. Modeling team member characteristics for the formation of a multifunctional team in concurrent engineering. IEEE Transactions on Engineering Management 51, 111-124.

Cowan, R., Jonard, N., Zimmermann, J.B., 2007. Bilateral collaboration and the emergence of innovation networks. Management Science 53, 1051-1067.

Dowlatshahi, S., 1999. A modeling approach to logistics in concurrent engineering. European Journal of Operational Research 115 (1), 59-76.

Deb, K., 1999. Multi-objective genetic algorithms: Problem difficulties and construction of test functions. Evolutionary Computation 7 (3), 205-230.

Deb, K., Agrawal, S., Pratap, A., Meyarivan, T., 2002. A fast and elitist multiobjective genetic algorithm: NSGA-II. IEEE Transactions on Evolutionary Computation 6 (2), 182-197.
Emden, Z., Calantone, R.J., Droge, C., 2006. Collaborating for new product development: Selecting the partner with maximum potential to create value. Journal of Product Innovation Management 23 (4), 330-341.

Fan, Z.P., Ma, J., Zhang, Q., 2002. An approach to multiple attribute decision making based on fuzzy preference information on alternatives. Fuzzy Sets and Systems 131 (1), 101-106.

Fan, Z.P., Ma, J., Jiang, Y.P., Sun, Y.H., Ma, L., 2006. A goal programming approach to group decision making based on multiplicative preference relations and fuzzy preference relations. European Journal of Operational Research 174 (1), 311321.

Fan, Z.P., Feng, B., Jiang, Z.Z., Fu, N., 2009. A method for member selection of R\&D teams using the individual and collaborative attribute. Expert Systems with Applications 36, 8313-8323.

Figueira, J., Greco, S., Ehrogott, M., 2005. Multiple Criteria Decision Analysis: State of the Art Surveys. Springer, New York.

Fitzpatrick, E.L., Askin, R.G., 2005. Forming effective worker teams with multifunctional skill requirements. Computers and Industrial Engineering 48 (3), 593-608.

Ford, R.C., Randolph, W.A., 1992. Cross-functional structures: A review and integration of matrix organization and project management. Journal of Management 18, 267-294.

Gloor, P.A., Paasivaara, M., Schoder, D., Willems, P., 2008. Finding collaborative innovation networks through correlating performance with social network structure. International Journal of Product Research 46 (5), 1357-1371.

Gulati, R., 1998. Alliances and networks. Strategic Management Journal 19, $293-$ 317.

Hacklin, F., Marxt, C., Fahrni, F., 2006. Strategic venture partner selection for collaborative innovation in production systems: A decision support systembased approach. International Journal of Production Economics 104, 100-112.

Haque, B., Pawar, K.S., Barson, R.J., 2000. Analysing organizational issues in concurrent new product development. International Journal of Production Economics 67, 169-182.

Hitt, M.A., Hoskisson, R.E., Nixon, R.D., 1993. A mid-range theory of interfunctional integration, its antecedents, and outcomes. Journal of Engineering and Technology Management 10, 161-185.

Holand, S., Gsaton, K., Gomes, J., 2000. Critical success factors for cross-functional teamwork in new product development. International Journal of Management Reviews 2 (3), 231-259.

Hwang, C.L., Yoon, K., 1981. Multiple Attribute Decision Making: Methods and Applications. Springer, Berlin.

Jozefowiez, N., Semet, F., Talbi, E.G., 2009. An evolutionary algorithm for the vehicle routing problem with route balancing. European Journal of Operational Research 195, 761-769.

Kaihara, T., Fujii, S., 2008. Game theoretic enterprise management in industrial collaborative networks with multi-agent systems. International Journal of Product Research 46 (5), 1297-1313.

Keller, R.T., 2001. Cross-functional project groups in research and new product development: Diversity, communications, job stress, and outcomes. Academy of Management Journal 44, 547-555.

Kuo, C.C., Glover, F., Dhir, K.S., 1993. Analyzing and modeling the maximum diversity problem by 0-1 programming. Decision Sciences 24, 1171-1185.

Lee, L.H., Chew, E.P., Teng, S.Y., Chen, Y.K., 2008. Multi-objective simulation-based evolutionary algorithm for an aircraft spare parts allocation problem. European Journal of Operational Research 189, 476-491.

Lee, L.H., Lee, C.U., Tan, Y.P., 2007. A multi-objective genetic algorithm for robust flight scheduling using simulation. European Journal of Operational Research 177 (3), 1948-1968.

Ma, J., Fan, Z.P., Huang, L.H., 1999. A subjective and objective integrated approach to determine attribute weights. European Journal of Operational Research 112 (2), 397-404.

Mansouri, S.A., 2005. A multi-objective genetic algorithm for mixed-model sequencing on JIT assembly lines. European Journal of Operational Research 167 (3), 696-716.

Msanjila, S.S., Afsarmanesh, H., 2008. Trust analysis and assessment in virtual organization breeding environments. International Journal of Product Research 46 (5), 1253-1295.

Nosratinia, A., Hunter, T.E., 2007. Grouping and partner selection in cooperative wireless networks. IEEE Journal on Selected Areas in Communications 25, 369378.

Orvosh, D., Davis, L., 1994. Using a genetic algorithm to optimize problems with feasibility constraints. Proceedings of the First IEEE Conference on Evolutionary Computation 2 (27-29), 548-553.

Saaty, T.L., 1980. The Analytic Hierarchy Process. McGraw-Hill Company, New York.

Schilling, M.A., Phelps, C.C., 2007. Interfirm collaboration networks: The impact of large-scale network structure on firm innovation. Management Science 53, 1113-1126.

Tan, K.C., Chew, Y.H., Lee, L.H., 2006. A hybrid multi-objective evolutionary algorithm for solving truck and trailer vehicle routing problems. European Journal of Operational Research 172, 855-885.

van de Water, T., van de Water, H., Bukman, C., 2007. A balanced team generating model. European Journal of Operational Research 180, 885-906.

Wang, Z., Yan, H.S., Ma, X.D., 2003. A quantitative approach to the organisation of cross-functional teams in concurrent engineering. International Journal of Advanced Manufacturing Technology 21, 879-888.

Zakarian, A., Kusiak, A., 1999. Forming teams: An analytical approach. IIE Transactions 31, 85-97. 